\title{
Geometry of the non-Abelian 2-index potential and twisted de Rham cohomology
}

\author{
TSOU Sheung Tsun* \\ and \\ Ioannis P. ZOIS ${ }^{\dagger}$ \\ Mathematical Institute, \\ 24-29 St. Giles', Oxford OX1 3LB, UK.
}

\begin{abstract}
It is found that the 2-index potential in nonabelian theories does not behave geometrically as a connection but that, considered as an element of the second de Rham cohomology group twisted by a flat connection, it fits well with all the properties assigned to it in various physical contexts. We also prove some results on the Euler characteristic of the twisted de Rham complex. Finally, provided that some conditions are satisfied, we propose a non-Abelian generalisation of S-duality.
\end{abstract}

PACS classification: 11.10.-z, 11.15.-q, 11.30.Ly

*tsou@ maths.ox.ac.uk

†izois@ maths.ox.ac.uk 


\section{The 2-index potential}

A skew rank 2 tensor field arises in various contexts: string theory, supergravity, and the loop space formulation of Yang-Mills theory. For notational convenience, we shall consider such a field $B_{\mu \nu}(x)$ interchangeably as a 2 -form over spacetime.

In the abelian case, the 2-index field is well studied [1] and fits neatly into the Dirac scheme of fields and potentials for general spin [2]. The field $B_{\mu \nu}(x)$ is usually regarded as a potential transforming under a gauge transformation $\Lambda_{\mu}(x)$ as

$$
\delta B_{\mu \nu}(x)=\partial_{\mu} \Lambda_{\nu}(x)-\partial_{\nu} \Lambda_{\mu}(x),
$$

exactly as say the electromagnetic potential but with one more index. One can also readily define the field strength, as a 3 -form field

$$
H_{\nu \rho \sigma}=\partial_{\sigma} B_{\nu \rho}+\partial_{\nu} B_{\rho \sigma}+\partial_{\rho} B_{\sigma \nu}
$$

The question immediately arises whether the $B_{\mu \nu}$ field can be interpreted as some sort of connection. This point was investigated by Teitelboim et al [3], and they found that one could regard such a 2-form as a parallel transport of loops (e.g. closed strings), provided the transformation is abelian, as in (1). But for nonabelian $B_{\mu \nu}$ we have to look elsewhere.

Freedman and Townsend [4 proposed a Lagrangian for the nonabelian $B_{\mu \nu}$. Cast in a first-order formulation of the non-linear $\sigma$ model, these fields appear as the dual of the Lagrange multiplier giving the flat connecton constraint, thus

$$
\mathcal{L}=\operatorname{Tr} A_{\mu} A^{\mu}+\operatorname{Tr}^{*} B_{\mu \nu} F^{\mu \nu},
$$

where ${ }^{*} B_{\mu \nu}=-\frac{1}{2} \epsilon_{\mu \nu \rho \sigma} B^{\rho \sigma}$ is the (Hodge) dual of $B_{\mu \nu}$, and Tr denotes the trace over the nonabelian indices. This action is invariant under the transformation

$$
\delta B_{\mu \nu}=D_{\mu} \Lambda_{\nu}-D_{\nu} \Lambda_{\mu}
$$

with $D_{\mu}=\partial_{\mu}-i g A_{\mu}$ the covariant derivative with respect to $A_{\mu}$, but $A_{\mu}$ itself should not transform.

A similar Lagrangian appears in a loop space formulation of Yang-Mills theory as a nonlinear $\sigma$ model [5, 6]:

$$
\mathcal{L}=\operatorname{Tr} \mathcal{A}_{\mu} \mathcal{A}^{\mu}+\operatorname{Tr}^{*} \mathcal{B}_{\mu \nu} \mathcal{F}^{\mu \nu}
$$


where $\mathcal{A}$ is the logarithmic derivative of the holonomy of the gauge potential $A$, and $\mathcal{F}$ the covariant curl of $\mathcal{A}$. This is in exact analogy with the Freedman-Townsend Lagrangian (3). Although the loop variables $\mathcal{A}_{\mu}$ can be thought of as a connection, under a Yang-Mills gauge transformation (which transforms the potential $A_{\mu}$ in the usual way), they are invariant. Moreover, the invariance of $\mathcal{L}$ under such a gauge transformation demands that the $\mathcal{B}$ field transforms as

$$
\delta \mathcal{B}_{\mu \nu}=\mathcal{D}_{\mu} \Lambda_{\nu}-\mathcal{D}_{\nu} \Lambda_{\mu}
$$

where $\mathcal{D}_{\mu}=\delta_{\mu}-i g \mathcal{A}_{\mu}$ is the loop covariant derivative corresponding to $\mathcal{A}_{\mu}$. This is exactly the Freedman-Townsend transformation (四). At the same time, this further confirms the result of [3] that nonabelian $B_{\mu \nu}$ does not behave like a connection, not even in loop space.

In this paper we shall present a geometric framework in which the $B_{\mu \nu}(x)$ field is not regarded as a gauge potential but as a cohomological freedom

intimately related to the existence of a flat connection $A_{\mu}$. We go on to explore further mathematical consequences of this construction which may have useful physical applications.

\section{Flat connections and the twisted de Rham complex}

For ease of presentation, in this section we shall use almost exclusively the index-free notation of differential forms.

By a flat connection we mean one with zero curvature. This means that we shall include the more general case where the base space $X$ (e.g. spacetime) need not be simply connected, in which case a flat connection may have nontrivial holonomy. In fact, it is well known that gauge equivalent classes of flat connections are in 1-1 correspondence with conjugacy classes of irreducible representations of $\pi_{1}(X)$ into the gauge group $G$ [7].

If we denote the exterior covariant derivative and curvature of the connection $A$ by $d_{A}$ and $F_{A}$ respectively, then on any form $\omega$ we have

$$
\begin{aligned}
d_{A}^{2} \omega & =d_{A}\left(d_{A} \omega\right) \\
& =d_{A}(d \omega+A \wedge \omega)
\end{aligned}
$$




$$
\begin{aligned}
& =d(d \omega+A \wedge \omega)+A \wedge(d \omega+A \wedge \omega) \\
& =(d A+A \wedge A) \wedge \omega \\
& =F_{A} \wedge \omega .
\end{aligned}
$$

Hence if $A$ is flat, $d_{A}^{2}=0$. This means that the exterior covariant derivative can actually be used as the differential in a differential complex, in direct contrast to the general Yang-Mills case.

Recall that associated to the principal $G$ bundle over $X$, with flat connection $A$, we have a flat vector bundle $E$ (with fibre the Lie algebra of $G$ ). We can consider the space $\Omega^{p}(X, E)$ of $p$-forms with values in $E$, which is by definition the space of global sections of the vector bundle $\left(\Lambda^{p} T^{*} X\right) \otimes E$, the tensor product of the $p$-th exterior power of the cotangent bundle $T^{*} X$ and the vector bundle $E$. Locally over an open set $U \subset X$ such a $p$-form is given by

$$
\omega=\sum \omega_{i} \otimes e^{i}
$$

where $\omega_{i}$ are $p$-forms on $U$ and $e^{i}$ are sections of $E$ over $U$, and the tensor product is over the algebra of $C^{\infty}$ functions on $U$. In our case of a flat vector bundle $E$, we can extend the usual de Rham complex $\Omega^{*}(X, d)$ over $X$ to a complex $\Omega^{*}\left(X, E, d_{A}\right)$ using the flat connection $A$. The flatness guarantees the existence of locally constant sections $e_{U}^{1}, \ldots, d_{U}^{n}(n=\operatorname{rank}$ of $E)$ with $d_{A} e_{U}^{i}=0$. We can then define the exterior derivative $d_{A} \omega$ of the form $\omega$ by

$$
d_{A}\left(\sum \omega_{e} \otimes e_{U}^{i}\right)=\sum\left(d \omega_{i}\right) \otimes e_{U}^{i}
$$

over the open set $U$. Since the sections $e_{U}^{i}$ are locally constant, it can readily be seen that $d_{A} \omega$ agrees on overlaps and hence is globally defined [ 8$]$. Moreover, $d_{A}^{2}=0$. It therefore makes sense to define the cohomology groups $H_{A}^{*}(X, E)$ as $d_{A}$-closed forms modulo $d_{A}$-exact forms in the usual way. It is easy to see that if $E$ is a trivial bundle of rank $n$ with the trivial flat connection, then $H_{\text {trivial }}^{*}(X, E)$ is just $n$ copies of the usual de Rham groups $H^{*}(X)$ (see [8] p.83 Proposition 7.4).

It is generally recognized that cohomology group elements correspond to physically interesting quantities [9]. If we now think of the $B$ field not as a 2 -from but as a representative of an element of $H^{2}$, then its transformation is nothing but the cohomological freedom of an exact 2-form:

$$
\delta B=d_{A} \Lambda
$$


with $\Lambda$ a 1-form, in other words, the transformation (14). It is therefore not a gauge freedom of the usual Yang-Mills type. Moreover, (10) reduces to (1) in the abelian case, which need not therefore be interpreted as a gauge (in the electromagnetism sense) transformation. In addition, the 3 -form $d_{A} B$ is as that discussed [10] for the 'curvature' of $B$.

As emphasized in [8] (see p.80 Example 7.1) and obvious from the definition (9), the cohomological groups depend in general on the particular trivialization chosen for $E$. This means that, if we think of $A$ as a connection in a principal bundle (as in the gauge case), then gauge equivalent $A$ 's may give rise to different $B$ 's. This makes perfect sense for the theory in hand, because the term $\operatorname{Tr} A^{2}$ in (3) makes it immediately obvious that the Lagrangian $\mathcal{L}$ is not 'gauge invariant'. This is why whereas $B$ transforms as in (田), $A$ must remain invariant.

The same observations apply to the loop space formulation of Yang-Mills theory. Since the phase factor is Yang-Mills gauge invariant, the loop space connection $\mathcal{A}$ is also gauge invariant. So is of course the Lagrangian in (5)) On the other hand, there is no freedom in transforming the loop connection $\mathcal{A}$, because that would mean changing the phase factor which is a physically measurable quantity.

The twisted de Rham cohomology groups $H_{A}^{*}(X, E)$ are topological invariants which are defined whenever there is a flat connection on a vector bundle $E$. Now a flat connection appears in many contexts which may be physically interesting, notably in integrable systems. This is not surprising: a flat connection ensures integrability of lifts. The following results are easy consequences of the definitions and may prove useful in studying the invariances of such systems.

In analogy with the usual Euler characteristic of a manifold, we make the following definition

Definition The Euler characteristic of the twisted de Rham complex

\footnotetext{
${ }^{1}$ Applying noncommutative geometry methods to the flat foliation induced by the flat connection $A$, one can study the $\eta$-invariant (related to global anomalies) which is more sensitive than the Euler characteristic defined here but less sensitive than the de Rham cohomology groups: it depends on the gauge equivalence class of $A$. This will be reported elsewhere [12].
} 
$\Omega^{*}\left(X, E, d_{A}\right)$ is defined to be

$$
\chi(X, E)=\sum_{i}(-1)^{i} \operatorname{dim}\left(H_{A}^{i}(X, E)\right) .
$$

The notation makes sense because of the following result.

Proposition Let $E$ be the adjoint vector bundle ad $P$, associated to the principal bundle $P$ over a manifold $X$ with structure group $G$ assumed compact and connected, equipped with a flat connection $A$. With respect to the induced connection $E$ is a flat vector bundle. Then the Euler characteristic $\chi(X, E)$ is independent of the flat connection used in calculating the cohomology groups $H_{A}^{i}(X, E)$.

Proof We shall prove this by calculating the Euler characteristic $\chi(X, E)$ using the symbol of an elliptic operator associated to the differential $d_{A}$.

For simplicity we shall use the same symbol $d_{A}$ for all the differentials in the complex:

$$
\left(d_{A}\right)_{p}:\left(\Lambda^{p} T^{*} X\right) \otimes E \rightarrow\left(\Lambda^{p+1} T^{*} X\right) \otimes E .
$$

We can 'assemble' the bundle (for details see [11]) by defining the single operator

$$
D_{d_{A}}: \Omega^{\text {even }} \rightarrow \Omega^{\text {odd }}
$$

where

$$
\begin{aligned}
\Omega^{\text {even }} & =\Gamma\left(\bigoplus_{p}\left(\Lambda^{2 p} T^{*} X\right) \otimes E\right), \\
\Omega^{\text {odd }} & =\Gamma\left(\bigoplus_{p}\left(\Lambda^{2 p+1} T^{*} X\right) \otimes E\right),
\end{aligned}
$$

defined by

$$
D_{d_{A}}=d_{A} \oplus d_{A}^{*}
$$

that is,

$$
D_{d_{A}}\left(\omega_{0}, \omega_{2}, \ldots\right)=\left(d_{A} \omega_{0}+d_{A}^{*} \omega_{2}, d_{A} \omega_{2}+d_{A}^{*} \omega_{4}, \ldots\right),
$$

where $d_{A}^{*}$ is the formal adjoint of $d_{A}$ with respect to some Riemannian metric on $X$.

Recall the symbol $\sigma(D)$ of a differential operator from sections of a vector bundle $E$ to sections of a vector bundle $F$

$$
D: \Gamma(E) \rightarrow \Gamma(F)
$$


is a vector bundle homomorphism

$$
\sigma(D): \pi^{*}(E) \rightarrow \pi^{*}(F)
$$

where, for $S X$ the unit sphere bundle in the tangent bundle, $\pi$ is the canonical projection $S X \rightarrow X$. In local coordinates, since $D$ is first order in this case, $\sigma(D)$ is obtained by replacing $\partial / \partial_{\mu}$ with $i \xi_{\mu}$, where $\xi_{\mu}$ is the $\mu$ th coordinate in the cotangent bundle $T^{*} X$. Furthermore, $D$ is elliptic if its symbol is invertible. This can be extended to a differential complex $E$ which is elliptic if the corresponding sequence of symbols $\sigma(E)$ is exact outside the zero section of $T X$.

For the flat bundle $E$, if we denote by $\Delta_{p}$ the Laplacian on $\Omega^{p}(X, E)$, thus

$$
\Delta_{p}=\left(d_{A}\right)_{p-1}\left(d_{A}^{*}\right)_{p-1}+\left(d_{A}^{*}\right)_{p}\left(d_{A}\right)_{p},
$$

then $\left(\right.$ since $\left.d_{A}^{2}=d_{A}^{* 2}=0\right)$

$$
\begin{aligned}
D_{A} D_{A}^{*} & =\bigoplus \Delta_{2 p} \\
D_{A}^{*} D_{A} & =\bigoplus \Delta_{2 p+1} .
\end{aligned}
$$

The exactness of the symbol complex $\sigma(\Omega(X, E))$ off the zero section then implies that $\sigma\left(\Delta_{p}\right)$ is an isomorphism (off the zero section). Therefore, $\Delta_{p}$ and hence $D_{A}$ are elliptic. Then it follows from the usual Hodge theory that

$$
\begin{aligned}
\operatorname{Ker} D_{A} & =\bigoplus h^{2 i} \\
\operatorname{Coker} D_{A} & =\bigoplus h^{2 i+1}
\end{aligned}
$$

where $h^{i}$ are the harmonic sections of the bundle $\bigoplus\left(\Lambda^{p} T^{*} X\right) \otimes E$, namely elements of Ker $\Delta^{i}$. This means we have found an elliptic operator $D_{A}$ whose index gives the required Euler characteristic:

$$
\operatorname{ind}\left(D_{A}\right)=\chi(X, E)
$$

By the Atiyah-Singer index formula [11], we know that this depends only on the symbol of $D_{A}$ and not on $D_{A}$ itself.

It is obvious from the above that the symbol of $d_{A}$ is independent of the flat connection $A$ used, since the term of highest degree is $\partial / \partial_{\mu}$. The symbol of $D_{A}$ is given by $i \xi-i \xi^{*}$ (where $\xi^{*}$ is contraction with $\xi$ ), also independent 
of $A$. The index of $D_{A}$ then gives the Euler characteristic as above, which is therefore independent of the flat connection $A$ used.

Corollary 1 In fact $\chi(X, E)=(\operatorname{dim} G) \times \chi(X)$.

Proof Obvious.

Corollary 2 When $X=\mathbf{R}^{4}, \chi(X, E)=\operatorname{dim} G$.

Proof Follows directly from Corollary 1.

Corollary 3 The Freedman-Townsend invariance actually corresponds to the stability equivalence relation in the definition of the topological $K$-groups.

Proof From the preceeding Proposition it is clear that since the principal symbol remains the same, we do not actually change our $K$-class. The Freedman-Townsend invariance is obviously not a change of the trivialisation of the bundle. Hence it must correspond to the second equivallence relation used in defining the $K$-groups, namely Grothendieck's stability relation.

\section{Remarks}

First we want to remark on a possible Non-abelian generalisation of S-duality.

It is clear that S-duality which is currently such an active field of research in theoretical physics, is actually a "Hodge star" duality between the field strengths of different gauge potentials. For example, the membrane/5-brane S-duality in M-Theory (see for example [17]) appears as follows: in general a p-brane moving in time sweps out a $(p+1)$-dim manifold, the worldvolume. This is described by a $(p+1)$-form denoted $B_{p+1}$ which is just the Poincare dual of the worldvolume. This $(p+1)$-form is seen as a gauge potential which gives rise to a field which is now a $(\mathrm{p}+2)$-form denoted $F_{p+2}$, which is defined to be

$$
F_{p+2}:=d B_{p+1}
$$

Then using the above notation one has for the membrane/5-brane duality in M-Theory that (recall that M-Theory has an 11-manifold as underlying space):

$$
F_{7}=* F_{4}
$$

Now remember that the Freedman-Townsend theory is exactly the above story for scalar fields (namely 0 -forms) but now $d$ is replaced by $d_{A}$, where 
$A$ is a flat connexion 1 -form. So essentially the point is that one can get a non-abelian generalisation of S-duality, to begin with, provided that one can have an analogue of the Hodge theory for the $d_{A}$-cohomology.

Assuming that our bundle also has a metric, a Hodge theory for the $d_{A^{-}}$ cohomology can be achieved if and only if the holonomy of the flat connection lies in the orthogonal group. So that can happen only for some flat connection 1-forms.

Now the second question is how can one get a 1-form to begin with and then constrain it via some equations of motion to become flat (just like in Freedman-Townsend case). In Freedman-Townsend case they were interested only in scalar fields and for the base space being actually $\mathbf{R}^{n}$, for some $n$, so they actually constructed the flat connection from the scalar field. The existence follows from the assumption that the coupling constant is small. But this is a very special case, what we actually need is a similar construction for a curved n-manifold and for arbitrary p-forms as gauge potentials.

We do not actually have something more specific on this, only two preliminary observations which are promising: the first is that a flat connection 1-form actually exists in the loop space formulation of the Chan-Tsou Dualised Standard Model (see [16] and [18]), so our idea to get a non-abelian generalisation of S-duality may be applied in that context.

As about M-Theory, there is actually a rather mysterious 1-form appearing in a purely topological Lagrangian for 5-branes in M-Theory using Gelfand-Fuchs cohomology [19]. Yet this has to be constrained somehow in order to become flat. This is all we can say for the moment. The interesting point here we believe is that in principle one can have an analogue of Hodge theory for the $d_{A}$-cohomology and that is really interesting.

In the case when $B_{\mu \nu}$ is abelian, it can be shown easily [4] that the theory is equivalent to a massless scalar field. This is an example of the general duality between scalar fields and $(d-2)$-form fields, where $d$ is the dimension of spacetime [14]. Here $d=4$. This duality also interchanges Bianchi identities (topology) and equations of motion (dynamics), reminiscent of the $\mathrm{Wu}$-Yang treatment of electric and magnetic charges [15, 6]. Similar considerations apply in the nonabelian case, giving the equivalence between the first-order and second-order formulations of the non-linear $\sigma$ model [4]. Here 
the scalar field is obtained from the flatness condition of $A_{\mu}$, which is locally of the form $g^{-1} \partial_{\mu} g$, with $g$ an element of the group $G$.

One may ask where the extra degrees of freedom of a spin 2 field have gone to, if it is equivalent to a scalar field. This is exactly accounted for by its cohomological freedom (10). Suppressing the Lie algebra indices, the 6 degrees of freedom of a skew rank 2 tensor are taken up by the 4 degrees of the vector $\Lambda_{\mu}$, plus its cohomological freedom of an additive scalar, leaving just the one degree of freedom of a scalar field.

In the case of Yang-Mills theory in loop space, this extra freedom gives rise to a dual gauge symmetry which is magnetic in nature if the original symmetry is considered to be electric. This leads to a fascinating electricmagnetic dual symmetry for Yang-Mills theory which is somewhat unexpected [16].

In conclusion, the interpretation of the 2-index field as a twisted de Rham cohomology group element, together with its inherent cohomological freedom, gives a geometric explanation of many of its properties. This is particularly interesting for the nonabelian case and may serve as a guide for studying its possible interactions. Furthermore, this geometric interpretation gives a satisfying picture for the loop space formulation of Yang-Mills theory, with particular regard to its symmetry properties.

The second author wishes to thank London Mathematical Society for financial support.

\section{References}

[1] K. Hayashi, Phys. Lett. 44B (1973) 497; M. Kalb and P. Ramond, Phys. Rev. D9 (1974) 2273; E. Cremmer andJ. Scherk, Nucl. Phys. B72 (1974) 117; Y. Nambu, Phys. Rept. 23 (1976) 250; J. Thierry-Mieg, Nucl. Phys. B335 (1990) 334; M. Blau and G. Thompson, Ann. Phys. 205 (1991) 137; P.J. Arias et al., Int. J. Mod. Phys. A4 (1992) 737.

[2] P.A.M. Dirac, Proc. Roy. Soc. London A155 (1936) 447.

[3] C. Teitelboim, Phys. Lett. B167 (1986) 63; M. Henneaux and C. Teitelboim, Found. Phys. 16 (1986) 593. 
[4] D.Z. Freedman and P.K. Townsend, Nucl. Phys. B177 (1981) 282.

[5] A.M. Polyakov, Nucl. Phys. B164 (1979) 171.

[6] Chan Hong-Mo, P. Scharbach and Tsou Sheung Tsun, Ann. Phys. (N.Y.) 167 (1986) 454.

[7] S.K. Donaldson and P.B. Kronheimer, The geometry of four manifolds (Oxford University Press, Oxford, 1990).

[8] See e.g. R. Bott and L.W. Tu, Differential Forms in Algebraic Topology (Springer-Verlag, New York, 1982).

[9] E. Witten, J. Diff. Geom. 17 (1982) 661.

[10] J. Thierry-Mieg and L. Baulieu, Nucl. Phys. B228 (1983) 259.

[11] M.F. Atiyah and I.M. Singer, Ann. Math. 87 (1968) 485.

[12] Ioannis P. Zois, D.Phil. thesis, Oxford University 1996; Tsou Sheung Tsun and Ioannis P. Zois, in preparation.

[13] P. Baum and R. Douglas, Proc. Symp. Pure Maths. 38 (AMS Publications, Rhode Island, 1982).

[14] E.S. Fradkin and A.A. Tseytlin, Ann. Phys. 162 (1985) 31; N.J. Hitchin et al, Comm. Math. Phys. 108 (1987) 535; E. Alvarez, L. Alvarez-Gaumé and Y. Lozano, Nucl. Phys. B424 (1994) 155.

[15] Tai Tsun Wu and Chen Ning Yang, Phys. Rev. D14 (1976) 437.

[16] Chan Hong-Mo, J. Faridani and Tsou Sheung Tsun, Phys. Rev. D53 (1996) 7293.

[17] M. J. Duff et all: "String Solitons", Phys. Rep. 259 (1995) 213 M. J. Duff: "Supermembranes", hep-th 9611203

[18] I. P. Zois: "On Polyakov's basic variational formulae on loop spaces", Rep. on Math. Physics 42. 3, (1998) 373. 
[19] I.P. Zois: "Gelfand Fuchs cohomology and M-theory as a TQFT", hepth 9906045 . 